\title{
CARACTERÍSTICAS E MEDIDAS ULTRASSONOGRÁFICAS DO BAÇO DE CÃES E GATOS FILHOTES HÍGIDOS
}

\section{ULTRASONOGRAPHIC CHARACTERISTICS AND MEASUREMENTS OF SPLEEN OF HEALTHY PUPPIES AND KITTENS}

\author{
Ivan F. Charas Santos ${ }^{1 *}$ \\ Maria Jaqueline Mamprim ${ }^{1}$ \\ Raquel Sartor ${ }^{2}$
}

\begin{abstract}
${ }^{1}$ Faculdade de Medicina Veterinária e Zootecnia da Universidade Estadual Paulista, Botucatu, SP, Brasil. ${ }^{2}$ Faculdade de Tecnologia de Botucatu, Botucatu, SP, Brasil.

*Autor para correspondência - ivansantos7@hotmail.com
\end{abstract}

\section{Resumo}

O objetivo do estudo foi analisar e comparar as características e as mensurações ultrassonográficas do baço entre cães e gatos filhotes hígidos, estabelecendo padrões de normalidade e valores de referência. Foram utilizados 15 cães e 15 gatos machos hígidos, raça mista e idade média de seis meses. Os mesmos foram divididos em dois grupos, sendo grupo G1 com 15 cães filhotes com peso médio de $3 \mathrm{~kg}$ e grupo G2 com 15 gatos filhotes com peso médio de $2 \mathrm{~kg}$. Os animais foram submetidos ao exame ultrassonográfico do baço para visibilização das características internas e mensuração do órgão. As características estruturais ultrassonográficas do baço foram analisadas e comparadas entre as duas espécies de forma descritiva por meio de imagens obtidas no exame. As mensurações foram comparadas estatisticamente usando-se o programa SPSS ${ }^{\circledR}$, com análise de variância (ANOVA), seguido do Teste de Tukey $(\mathrm{P}<0,05)$. Em ambos os grupos, foi observado que as artérias esplênicas apresentaram menor diâmetro luminar em relação às veias esplênicas. Nos cães e gatos filhotes, a ecotextura foi visibilizada homogênea e finamente granular. O baço dos cães filhotes tende a ser maior em comprimento $(6,87 \pm 0,03 \mathrm{~cm})$ e largura $(5,95 \pm 0,07 \mathrm{~cm})$ em relação ao comprimento $(4,53 \pm 0,02 \mathrm{~cm})$ e largura $(4,55 \pm 0,04)$ nos gatos filhotes. Concluiu-se que não existe diferença de ecotextura e ecogenicidade esplênica entre o cão filhote e gato filhote e o baço dos cães filhotes apresentrou dimensões maiores, em comprimento e largura, em relação aos gatos filhotes.

Palavras-chaves: baço; ecogenecidade; mensuração pequenos animais; ultrassom.

\begin{abstract}
The aim of the study was to analyze and compare the characteristics and ultrasound measurements of the spleen of healthy puppies and kittens, establishing normal standards and landmarks. We used 15 healthy male dogs and 15 healthy male cats, mixed breed and average age of six months. They were divided into two groups: G1 with 15 puppies with an average weight of $3 \mathrm{~kg}$ and G2 with 15 kittens with an average weight of $2 \mathrm{~kg}$. The animals underwent ultrasound examination of the spleen for visualization of internal characteristics and measurement of the organ. The structural characteristics was analyzed and compared between the two species through the images obtained
\end{abstract}


in the exam. The measurements were compared statistically using the SPSS program, with analysis of variance (ANOVA) followed by Tukey test $(\mathrm{P}<0.05)$. In both groups, we observed the splenic artery showed smaller diameter than the splenic veins. In puppies and kittens, the echotexture was visualized homogeneous and finely granular. The puppy's spleen tends to be bigger in length (6.87 $\pm 0.03 \mathrm{~cm})$ and width $(5.95 \pm 0.07 \mathrm{~cm})$ in relation to spleen length $(4,53 \pm 0,02 \mathrm{~cm}))$ and width $(4.55 \pm 0.04)$ in kittens. We concluded there is no difference of echotexture and splenic echogenicity between puppies and kittens, and the spleen of puppies showed bigger dimensions than in kittens. Keywords: echogenicity; size; small animals; spleen; ultrasound.

Enviado em: 18 janeiro 2016

Aceito em: 12 de setembro de 2016

\section{Introdução}

O exame ultrassonográfico é utilizado como um método complementar no diagnóstico de imagem de referência para a visibilização esplênica, sendo de baixo custo e mais acessível em relação à tomografia e à ressonância magnética ${ }^{(1)}$. A ultrassonografia possibilita a avaliação estrutural do baço e da dinâmica vascular ${ }^{(2)}$.

O exame ultrassonográfico do baço pode ser realizado com o animal em posição de decúbito dorsal, decúbito lateral direito ou em estação ${ }^{(2)}$. Em cães e gatos, o baço pode ser localizado entre o primeiro e segundo espaço intercostal esquerdo, estendendo-se em direção caudal em relação ao fígado, lateralmente em relação ao estômago e a sua extremidade livre localiza-se dorsalmente à bexiga ${ }^{(2,3)}$. O baço é examinado nos cortes sagital e transversal ${ }^{(4)}$. A ecotextura é visibilizada homogênea, finamente granular, sendo mais densa que o parênquima hepático e gordura do ligamento falciforme. Observa-se hiperecogênico em relação ao córtex renal e parênquima hepático e hipoecogênico em relação à gordura do seio renal ${ }^{(2,4)}$.

As mensurações dos diversos órgãos abdominais em pequenos animais são limitadas pela falta de definição dos bordos dos mesmos e pela dificuldade de se obter planos seccionais completos e presença de $\operatorname{artefatos}^{(5)}$. Desse modo, a dimensão do baço é considerada subjetiva e a sua mensuração é realizada por meio da radiografia, tomografia computadorizada e ressonância magnética ${ }^{(2,4)}$.

Do ponto de vista clínico, o exame ultrassonográfico abdominal identifica alterações quer no seu estágio inicial ou avançado. Assim, associado aos escassos relatos sobre o padrão estrutural e mensurações ultrassonográficas do baço em cães e gatos filhotes e à necessidade de se estabelecer padrões de normalidade esplênica nos mesmos, o objetivo deste estudo é analisar e comparar as características e as mensurações ultrassonográficas do baço entre cães e gatos filhotes hígidos, estabelecendo padrões de normalidade e valores de referência.

\section{Material e Métodos}

O estudo foi aprovado pela comissão de Ética no Uso de Animais em Experimentação da Faculdade de Medicina Veterinária e Zootecnia - CEUA/ FMVZ-UNESP-Botucatu, com número de protocolo 081/2009.

Quinze cães e 15 gatos machos hígidos, sem raça determinada (SRD) e com idade média de seis meses (5 - 6 meses) foram inclusos no estudo. Os mesmos foram divididos em dois grupos: grupo G1 - 15 cães filhotes com peso médio de $3 \mathrm{~kg}(3-4 \mathrm{~kg})$; grupo G2 - 15 gatos filhotes com peso médio 
de $2 \mathrm{~kg}(1-3 \mathrm{~kg})$. A higidez foi determinada pelo exame físico, eritrograma, leucograma, contagem plaquetária, bioquímica sanguínea sérica (alanina aminotransferase - ALT, ureia e creatinina), urinálise completa (densidade específica, sedimento urinário e teste dipstick) e exame de fezes. Foi realizado exame ultrassonográfico abdominal de todos os animais. O critério de inclusão para os cães e gatos filhotes foi a presença de higidez.

Todos os animais foram submetidos à privação de alimentos sólidos durante quatro a cinco horas, tiveram acesso livre à água e receberam dimeticolina (Dimeticol ${ }^{\circledR}$, Hipolabor Farmacêutica, São Paulo), na dose de $9,7 \mathrm{mg} / \mathrm{kg}, \mathrm{PO}$, a cada oito horas antes da realização do exame. Os animais foram contidos fisicamente por duas pessoas e colocados em decúbito lateral direito, sem qualquer tipo de sedação ou tranquilização. Foi realizada uma tricotomia ampla do abdômen, abrangendo o sétimo espaço intercostal e região do osso púbis e lateralmente até a região proximal dos processos transversos das vértebras lombares. Álcool isopropílico e gel acústico foram utilizados para evitar a presença de ar entre a pele e o transdutor.

Os exames ultrassonográficos foram realizados utilizando-se o aparelho GE-Logic 3 (Gel Ultra®, Parker Laboratories Inc., USA), equipados com transdutores convexo de 3,5 - 5,0 MHz e transdutor linear de $80 \mathrm{~mm}$ de comprimento (resolução axial de $<1,1 \mathrm{~mm}$, resolução lateral de $<0,9 \mathrm{~mm}$, profundidade de $2 \mathrm{~cm}$ ), frequência de $6-10 \mathrm{MHz}$, no modo B. Foram realizados cortes sagital e transversal para identificação das características ultrassonográficas e mensurações do baço. $\mathrm{O}$ ponto de referência anatômico para visualização do baço foi a região imediatamente caudal ao arco costal esquerdo. O Doppler foi utilizado para acessar o fluxo sanguíneo das artérias e veias lienais, utilizadas também como pontos de referência para identificação do hilo esplênico.

O tempo de realização do exame ultrassonográfico do baço em ambos os grupos foi cronometrado utilizando-se um cronometro digital de precisão.

Os animais foram colocados em decúbito lateral direito e o transdutor foi posicionado na região caudal do arco costal esquerdo, ventralmente aos músculos longos dorsais, sendo identificada a margem caudal do baço, crâniolateral ao rim esquerdo. Uma vez identificada a margem caudal do baço, o transdutor foi rotacionado em $90^{\circ}$ e a margem cranial do baço foi localizada, apresentando uma forma triangular e arredondada.

As características estruturais e dimensões ultrassonográficas do baço foram analisadas e comparadas entre as duas espécies de forma descritiva por três especialistas da área. Para tal, foram utilizadas gravações das imagens obtidas no dia do exame.

Devido à falta de critério de mensuração do baço e à impossibilidade de se realizar uma imagem de todo o órgão, o mesmo foi dividido em duas regiões para mensuração da largura do baço: da margem cranial e da margem caudal ao hilo lienal. A largura do baço em cães filhotes foi determinada como sendo a soma das distâncias entre as margens cranial e caudal ao hilo lienal (Figura 1A e 1B). Nos gatos filhotes, a largura do baço foi determinada como sendo a soma da distância entre a margem cranial e caudal do baço (Figura 1C). Nos cães e gatos filhotes, o ponto de referência para a mensuração do comprimento do baço foi determinado pelo hilo lienal. O comprimento foi determinado como sendo a distância ente as extremidades dorsal e ventral do baço (Figura 1D).

As mensurações foram comparadas estatisticamente usando o programa SPSS ${ }^{\circ}$, com análise de variância (ANOVA), seguido do Teste de Tukey $(\mathrm{P}<0,05)$. 


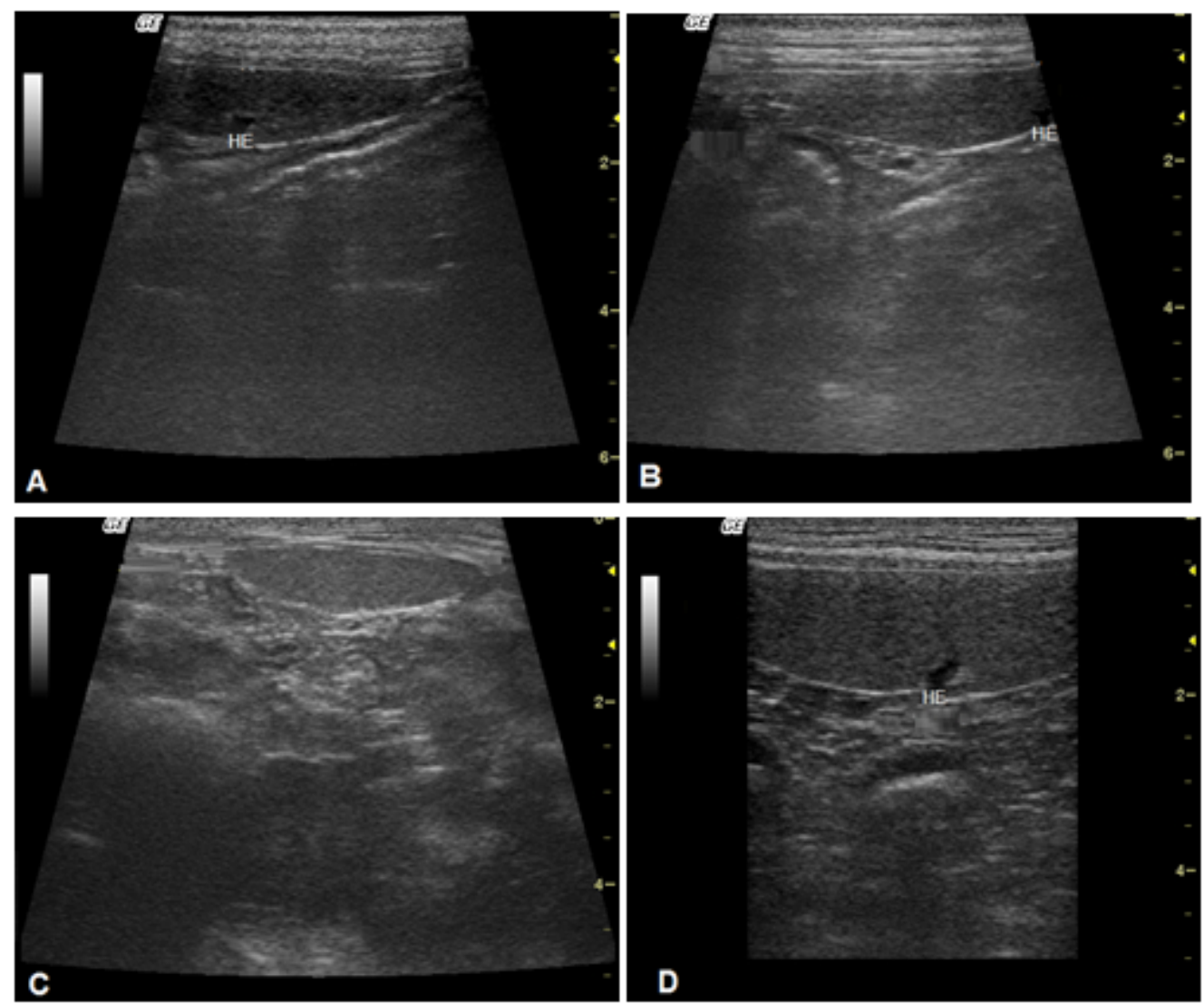

Figura 1: Sonograma ( $7,5 \mathrm{HZ}$, modo $\mathrm{B}$, corte sagital) do baço de cão filhote, ilustrando a mensuração da largura do baço, determinada pela soma da distância entre a margem caudal e o hilo lienal (HE) (A) e a distância entre a margem cranial e o $\mathrm{HE}$ (B). Sonograma $(7,5 \mathrm{HZ}$, modo $\mathrm{B}$, corte transversal) do baço de gato filhote, ilustrando a mensuração da largura do baço, determinada pela distância entre a margem caudal e cranial (C). Sonograma $(7,5 \mathrm{HZ}$, modo B, corte sagital) do baço de cão filhote, ilustrando a mensuração do comprimento do baço, determinado pela distância entre as extremidades dorsal e ventral do baço (D).

\section{Resultados}

Em ambos os grupos, o baço foi visibilizado utilizando-se o transdutor linear na frequência de 7,5 $\mathrm{MHz}$, no modo B, nos cortes transversal e sagital, sendo que o mesmo foi identificado na região lateral esquerda do abdômen - fossa paralombar esquerda, caudalmente ao arco costal e cranialmente ao rim esquerdo. Com o uso do Doppler, foi observado que as artérias lienais apresentaram menor diâmetro do lúmen em relação às veias lienais e as paredes visibilizadas apresentaram-se hiperecogênicas, em ambos os grupos. As veias lienais foram visibilizadas em forma de $\mathrm{Y}$ ao penetrar no hilo lienal e lúmen de conteúdo anecogênico, tornando-se visíveis dentro do parênquima esplênico.

O tempo médio e desvio padrão do exame ultrassonográfico do baço nos cães filhotes foi de $12 \pm 1$ minuto e nos gatos filhotes foi de $9 \pm 1$ minuto. Nos cães e gatos filhotes, a ecotextura foi visibilizada homogênea, finamente granular, sendo o baço visibilizado hiperecogênico em relação ao córtex renal e parênquima hepático e a cápsula esplênica hiperecogênica. Nos cães filhotes, o hilo lienal com conteúdo anecogênico foi observado no corte sagital e transversal e nos gatos filhotes somente no corte sagital (Figura 2).

As mensurações da largura e comprimento do baço dos cães e gatos filhotes foram ilustradas na Tabela 1. 

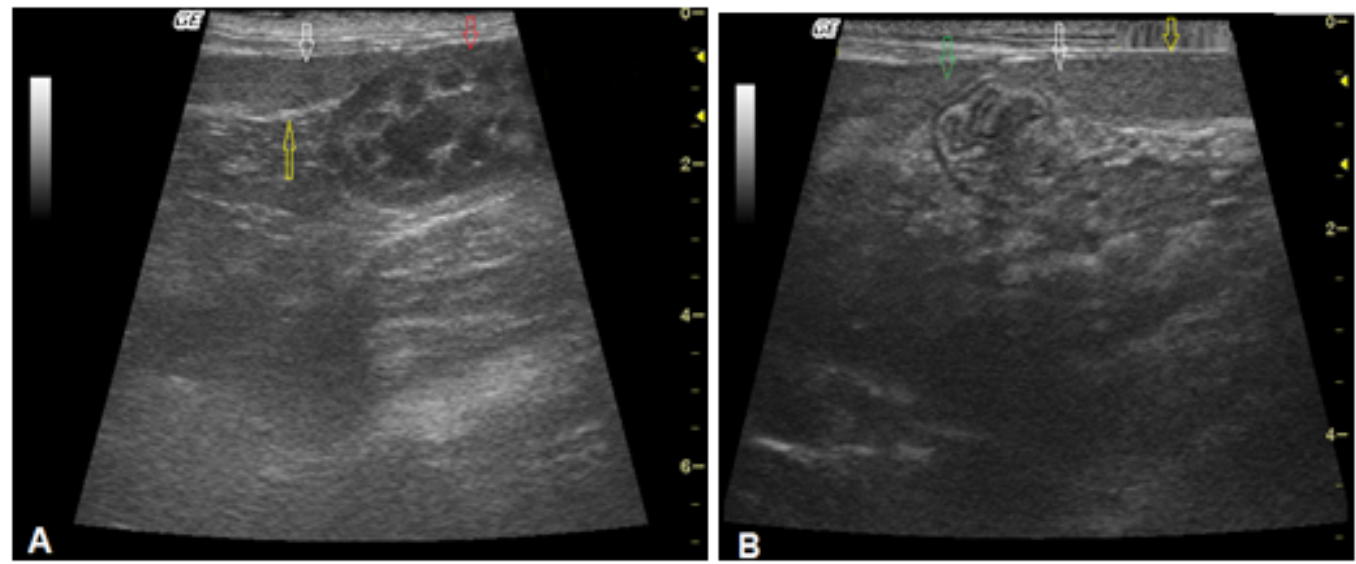

Figura 2: Sonograma ( $7,5 \mathrm{HZ}$, modo $\mathrm{B}$, corte sagital) de gato filhote com idade média de seis meses e $2 \mathrm{~kg}$ de peso corpóreo médio, ilustrando o baço (seta branca) com ecotextura homogênea e finamente granular, cápsula esplênica hiperecogênica (seta amarela), hiperecogenicidade em relação ao córtex renal (seta vermelha) (A) e hiperecogenicidade em relação ao parênquima hepático (seta verde) (B).

Tabela 1: Mensurações (cm) da largura e comprimento do baço dos cães filhotes (G1) e gatos filhotes (G2) (média \pm desvio padrão)

\begin{tabular}{lcc}
\hline Grupos & G1 $^{\boldsymbol{*}}$ & G2 $^{\boldsymbol{*}}$ \\
\hline Largura & $6,90 \pm 0,03^{\mathrm{a}}$ & $4,50 \pm 0,02^{\mathrm{b}}$ \\
Comprimento & $0,59 \pm 0,07^{\mathrm{a}}$ & $0,46 \pm 0,04^{\mathrm{b}}$ \\
\hline
\end{tabular}

Médias seguidas por letras diferentes, na mesma linha e mesma coluna, diferem entre si pelo teste de Tukey $(\mathrm{P} \approx 0,05) \mathrm{e}$ estabelecem a comparação entre os grupos ( $\mathrm{n}=15)$.

Foram observadas diferenças significativas da largura e comprimento do baço entre os cães filhotes e gatos filhotes, sendo que os cães filhotes apresentaram largura e comprimento maior em relação os gatos filhotes.

\section{Discussão}

As características ultrassonográficas normais dos órgãos abdominais em cães adultos já foram descritos e os padrões de referência estabelecidos ${ }^{(6-7)}$. Entretanto, relatos sobre características e mensurações ultrassonográficas de órgãos abdominais em cães e gatos filhotes são escassos na literatura, excetuando-se os relatos em relação ao rim $^{(8)}$ e glândulas adrenais ${ }^{(9)}$. Contudo, existe a necessidade de que o ultrassonografista saiba identificar os padrões de normalidade ultrassonográficos (características e dimensões) do baço nos cães e gatos filhotes para posteriormente identificar as possíveis alterações esplênicas.

Estudos prévios demonstraram que as mensurações dos órgãos abdominais são influenciadas pelo sexo, idade e peso corpóreo ${ }^{(10-11)}$. Desse modo, os animais do presente estudo foram padronizados de acordo com o sexo, peso e idade. Não foi possível padronizar em relação à raça devido ao baixo número de gatos de raça na região.

Segundo Stander et al. ${ }^{(12)}$, o exame ultrassonográfico de pacientes pediátricos sem contenção química foi difícil de ser realizado devido à relutância dos mesmos a manterem-se estáveis durante os 20 minutos do exame, diferentemente do presente estudo, visto que o método de contenção dos animais foi satisfatório para a realização dos exames ultrassonográficos do baço, que duraram em média 12 minutos para os cães filhotes e 9 minutos para os gatos filhotes.

No presente estudo, foi utilizado um transdutor linear de 7,5 MHz, no modo B, para visibilização do 
baço em ambos os grupos, coerente com a literatura ${ }^{(4)}$. Porém, também, é possível utilizar transdutores convexos de $10 \mathrm{MHz}^{(4)}$. Santos et al. ${ }^{(8)}$ e Santos et al. ${ }^{(9)}$ utilizaram transdutores lineares de 7,5 MHz para a visibilização das glândulas adrenais e do rim em cães e gatos filhotes saudáveis, respectivamente.

De acordo com Nyland e Hager ${ }^{(4)}$ e Scholz e Luerssen ${ }^{(13)}$, o baço é examinado nos cortes sagital e transversal e com os animais em posição de decúbito lateral direito, similar ao presente estudo. Porém, o hilo lienal dos gatos filhotes somente foi observado no corte sagital. A posição do transdutor do lado esquerdo da parede abdominal, caudalmente ao arco costal e ventralmente aos músculos longos dorsais foi de acordo com o citado pela literatura ${ }^{(4,13)}$. Nyland et al. ${ }^{(13)}$ relataram a possibilidade de realização do exame ultrassonográfico do baço em gatos na posição de decúbito dorsal e lateral direito em estação.

A posição do baço, localizado superficialmente no abdômen, facilitou a sua visibilização, não havendo interferência da interposição do gás intestinal eventualmente presente em alguns animais ${ }^{(2,13)}$. No presente estudo, não foi observada a presença de fluido peritoneal nos cães e gatos filhotes. Stander et al. ${ }^{(12)}$ realizaram um estudo ultrassonográfico com 33 cães filhotes da raça Beagle e relataram a presença de pequena quantidade de fluido peritoneal anecóico. Os mesmos autores consideraram normal a presença dessa pequena quantidade de fluido peritoneal, visto que o mesmo possuía baixa celularidade.

Segundo Nyland et al. ${ }^{(13)}$, o baço dos gatos possui ecogenicidade e ecotextura similar ao do cão, o mesmo observado no presente estudo em filhotes. A ecotextura apresentou-se homogênea e fina e hiperecogênica em relação ao parênquima hepático e córtex renal ${ }^{(2,4,13)}$. As principais diferenças entre as artérias e veias lienais (diâmetro e hiperecogenicidade) dos cães e gatos filhotes foram similares aos achados da literatura ${ }^{(4,13)}$.

A dimensão do baço é considerada subjetiva na Medicina Veterinária. Segundo Baker e Davidson ${ }^{(2)}$ e Nyland e Hager ${ }^{(4)}$, a presença de esplenomegalia desloca o intestino delgado para a direita ou para a esquerda, como também desloca o estômago cranialmente. No cão, o tamanho do baço é muito variável e sua mensuração deve ser relacionada com o exame radiográfico ${ }^{(4)}$. $\mathrm{Na}_{\text {medicina humana não }}$ existe critério ultrassonográfico absoluto sobre mensuração do baço. Quando o mesmo se encontra normal, pode apresentar o tamanho aproximado do rim esquerdo, sendo que o seu comprimento não pode passar $15 \mathrm{~cm}^{(14)}$.

No presente estudo, não se utilizou o exame radiográfico como meio auxiliar na mensuração do baço $^{(4)}$, visto que o objetivo do trabalho foi a determinação das dimensões ultrassonográficas.

Não havendo uma metodologia de mensuração ultrassonográfica do baço em cães filhotes, o baço foi dividido em duas regiões (margens cranial e caudal ao hilo lienal) e suas mensurações foram somadas para se obter a largura do baço. Por outro lado, em gatos filhotes não foi necessário dividir o baço em regiões, visto que foi possível visibiliza-lo em uma única imagem.

As diferenças de tamanho em relação à largura e ao comprimento do baço entre os cães e gatos filhotes poderão estar relacionadas com a diferença de conformação corporal entre ambos, visto que foi observada uma diferença significativa entre os pesos dos cães e gatos filhotes com idades similares. Por outro lado, a metodologia utilizada para mensurar o baço dos cães filhotes pode influenciar na diferença de tamanho esplênico entre as espécies. Santos et al. ${ }^{(8)}$ relataram que a diferença na comparação das dimensões renais entre cães e gatos filhotes com peso e idade similares poderia estar relacionada com a diferença de conformação entre as espécies.

\section{Conclusões}

De acordo com a metodologia usada, pode-se concluir que não existe diferença de ecotextura e ecogenicidade esplênica entre o cão filhote e gato filhote. $O$ baço dos cães filhotes são maiores, em largura e comprimento, em relação ao dos gatos filhotes. 


\section{Agradecimentos}

À Fundação Ford pela bolsa de mestrado.

\section{Referências}

1. Debruyn K, Haers H, Combes A, Paepe D, Peremans K, Vanderperren k, et al. Ultrasonography of the feline kidney technique, anatomy and changes associated with disease. Clinical review. J Fel Med Surg. 2012; 14: 231-6.

2. Baker TW, Davidson AP. Pediatric abdominal ultrasonography. Vet Clin North Am: Small Anim Pract. 2006; 36: 641-655.

3. Hecht, S. Spleen. In: Pennick D, Anjo MA. Atlas of small animal ultrasonography. 2 ed. Iowa: Blackwell Publishing; 2015. 263.

4. Nyland TG, Hager DA. Sonography of the liver, gallbladder, and spleen. Vet Clin North Am Small Anim Pract. 1985; 15: 1123-1148.

5. Barr FJ, Holt PE, Gibbs C. Ultrassonographic measurement of normal renal parameters. J Small Anim Prac. 1990; 31: 180-4.

6. Penninck DG, Nyland TG, Fisher PE, Kerr LY. Ultrasonography of the normal canine gastrointestinal tract. Vet Rad. 1989; 30: 272-6.

7. Delaney F, O'Brien RT, Waller K. Ultrasound evaluation of small bowel thickness compared to weight in normal dogs. Vet Rad Ultrasound. 2003; 44: 577-580.

8. Santos IFC, Mamprim MJ, Sartor R. Comparação das características e medidas ultrassonográficas das glândulas adrenais de cães e gatos filhotes saudáveis. Ciênc Anim Bras. 2013; 14(4): 514-521.

9. Santos IFC, Mamprim MJ, Sartor R. Comparação das características e medidas ultrassonográficas do rim entre cães e gatos filhotes. Rev Bras Med Vet. 2015; 37(3): 186-192.

10. Seyrek-Intas D, Kramer M. Renal imaging in cats. Vet Focus. 2008; 18: 23-30.

11. Park I, Lee H, Kim J, Nam S, Choi R, Oh K. Ultrasonographic evaluation of renal dimension and resistive index in clinically healthy Korean domestic short-hair cats. J Vet Scien. 2009; 9: 415-9.

12. Stander N, Wagner WM, Goddard A, Kirberger RM. Normal canine pediatric gastrointestinal ultrasonography. Vet Rad Ultrasound. 2010; 51(1): 75-78.

13. Nyland TG. Baço. In: Nyland TG, Mattoon JS. Ultra-som diagnóstico em pequenos animais. 2 ed. São Paulo: Roca; 2005. 131-138.

14. Safak AA, Simsek E, Bahcebasi T. Sonographic assessment of the normal limits and percentile curves of liver, spleen, and kidney dimensions in healthy school-aged children. J Ultrasound Med. 2005; 24: 13591364. 